\title{
How to HaNDL This Case?
}

\author{
Filipa Silva $^{\mathrm{a}, \mathrm{c}}$, Rita Ferraz ${ }^{\mathrm{b}}$, Nelia Neves $^{\mathrm{b}}$, Paulo Andrade ${ }^{\mathrm{b}}$, Claudia Carvalho ${ }^{\mathrm{b}}$, Antonio Sarmento ${ }^{\mathrm{b}}$
}

\begin{abstract}
Headache and neurological deficits with cerebrospinal fluid (CSF) lymphocytosis (HaNDL) is a self-limited, benign neurological entity that may constitute a diagnosis dilemma since it can mimic a variety of conditions including aseptic meningitis, meningoencephalitis, migraine with aura and stroke. We report a case of a 31-year-old Caucasian man admitted at the emergency department for sudden onset of dysarthria and paresthesias in the right upper limb, after 2 weeks of complaints of headache associated with mild nausea. After extensive blood investigation, CSF study, electroencephalogram and neuroimaging, central nervous system infections, autoimmune and vascular diseases were excluded. CSF study revealed lymphocytic pleocytosis, normal glucose and slightly elevated protein concentration. Magnetic resonance imaging (MRI) showed a diffuse leptomeningeal enhancement, a decreased and delayed perfusion to the entire left hemisphere. The patient improved with supportive and symptomatic management. Two days after discharge, he developed a similar clinical picture and recovered completely 30 minutes after admission. His repeated lumbar puncture (LP) showed progressive improvement as expected due to the clinical resolution and MRI did not reveal any of the previous changes. Given the presentation (clinical picture, imaging and CSF studies), the subsequent similar and self-limited episode and having excluded other causes, a final diagnosis of HaNDL was made. HaN$\mathrm{DL}$ is one of the most frequent stroke mimics and probably not as rare as commonly thought. It has a good prognosis since this monophasic, self-limiting condition runs its course usually within a few months from onset, without any neurological sequelae.
\end{abstract}

Keywords: HaNDL; Headache; Stroke; Lymphocytic pleocytosis; Cerebrospinal fluid; Cerebral blood flow; MRI

\section{Introduction}

Headache and neurological deficits with cerebrospinal fluid

Manuscript accepted for publication January 20, 2016

${ }^{a}$ Internal Medicine Department, Centro Hospitalar de Sao Joao, Porto, Portugal

bInfectious Disease Department, Centro Hospitalar de Sao Joao, Porto, Portugal

${ }^{\mathrm{c}}$ Corresponding Author: Servico de Medicina Interna, Centro Hospitalar de Sao Joao, E.P.E., Alameda Prof. Hernani Monteiro, 4200-319 Porto, Portugal. Email: filipadcs@gmail.com

doi: http://dx.doi.org/10.14740/jmc2419w
(CSF) lymphocytosis (HaNDL), also known as pseudomigraine with lymphocytic pleocytosis, is a self-limited, benign neurological entity that was first described in the early 1980 s by Swanson and Marti-Masso [1,2]. This syndrome may constitute a diagnosis dilemma since it has a diverse spectrum of clinical manifestations, its etiology is not fully understood and it can mimic a variety of conditions including aseptic meningitis, meningoencephalitis, migraine with aura and stroke.

The syndrome is a triad of transient focal neurological deficits, headache and lymphocytic pleocytosis. Knowledge about this disorder is important because its self-limited nature and favorable outcome make other therapeutic interventions, besides symptomatic treatment, largely unnecessary [3].

\section{Case Report}

A 31-year-old Caucasian man, an employee in a transport company, was asymptomatic until 2 weeks prior to admission when he started complaints of moderate continuous, holocranial, pulsating headache associated with mild nausea, without other symptoms. On the second week of headache, he recalled sudden onset of dysarthria and paresthesias in the right upper limb and face and was admitted at the emergency department (ED). Besides the smoking habits (0.5 packs per day), past medical history was irrelevant, and he denied hypertension, diabetes mellitus, trauma, alcohol and illicit drug use.

On the initial evaluation at ED, his vital signs were normal and he was conscious, with dysarthria and right arm paresis (manual muscle test score 4/5) without any other deficits or signs of meningeal irritation. He underwent a brain CT scan that excluded any ischemic or hemorrhagic lesions. While at the ED his clinical condition rapidly deteriorated: obnubilation, aphasia, right hypoesthesia (NIHSS 10), so it was decided to perform thrombolysis with alteplase. Despite treatment, the patient did not recover deficits and within $1 \mathrm{~h}$ an episode of tonic movements and myoclonus was documented. CT angiogram was performed and no significant changes were present. He was started on antiepileptics, ceftriaxone, ampicilin and acyclovir. Lumbar puncture (LP) had been delayed for $24 \mathrm{~h}$, since he had recently performed thrombolysis.

Workup revealed normal blood count. Serum electrolytes and renal and liver function tests were also within normal range, inflammatory markers were low (PCR $24.3 \mathrm{mg} / \mathrm{L}$ ), urine toxicology screen was negative, and X-ray and urine analysis were unremarkable. In less than $12 \mathrm{~h}$ since the onset of the symptoms, he had a complete reversal of all deficits, and eventually became asymptomatic in 3 days. Electroencephalogram (ECK) was normal and magnetic resonance imaging (MRI) 

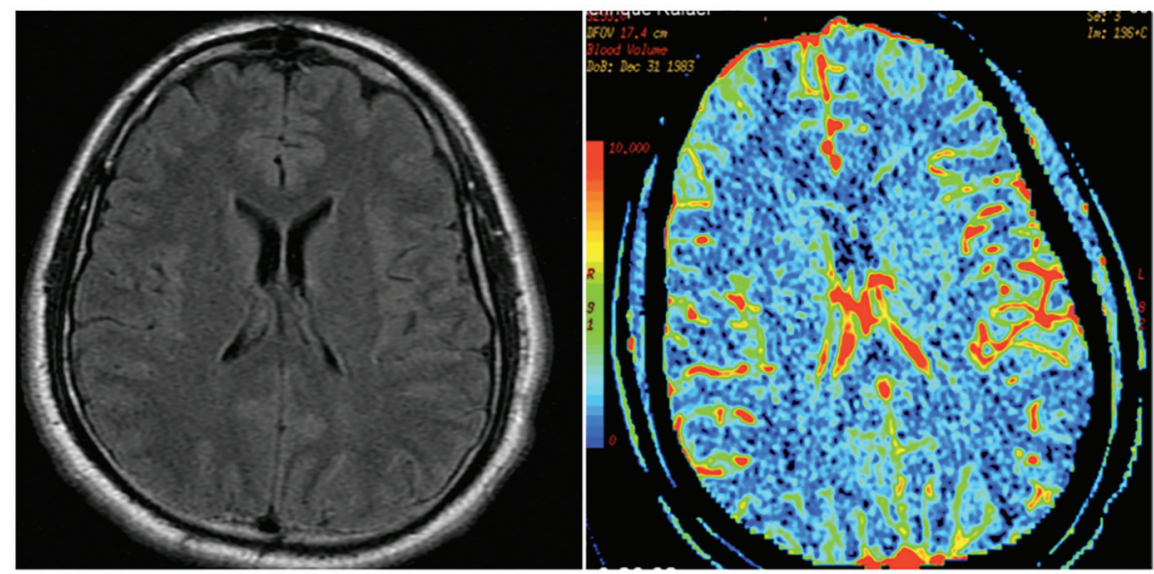

(A)

(B)
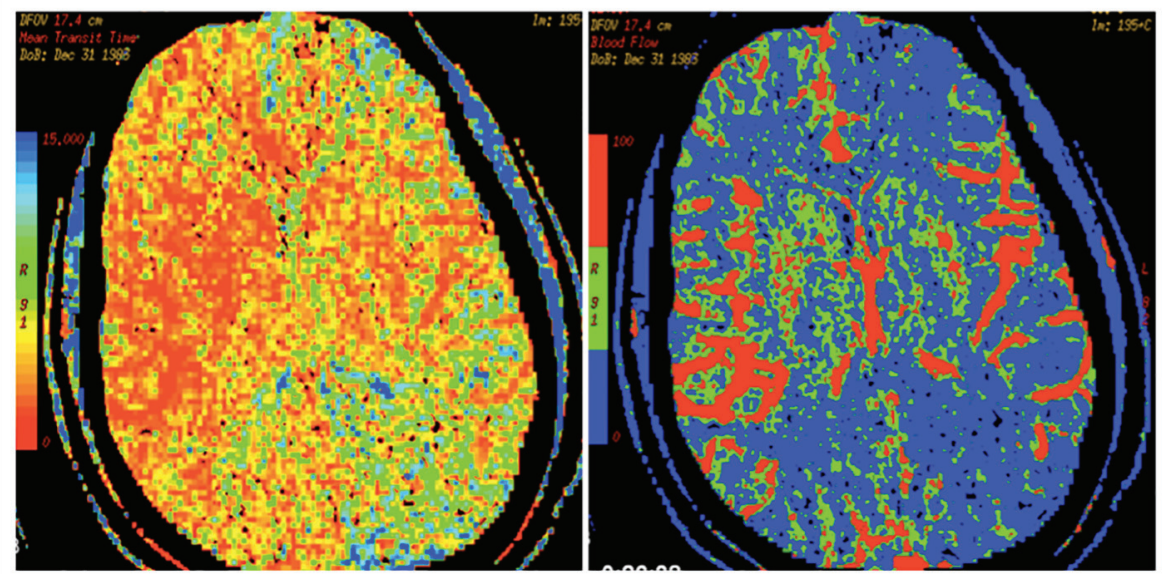

(C)

(D)

Figure 1. Axial images reveal (A) normal MRI, (B) normal cerebral blood volume in $C T,(C)$ prolonged $C T$ mean transit time to the entire left hemisphere relative to the right at presentation, and (D) reduction in cerebral blood flow.

revealed a diffuse leptomeningeal enhancement, a decreased and delayed perfusion to the entire left hemisphere (Fig. 1). LP revealed 484 cells with mononuclear predominance $(98 \%)$, normal glucose $(0.74 \mathrm{mg} / \mathrm{L})$ and slightly elevated protein concentration $(0.52 \mathrm{~g} / \mathrm{L})$. The opening pressure was not measured. CSF polymerase chain reaction (PCR) for HSV1 and 2, EBV, CMV, Varicella-Zoster, Enterovirus, Mycoplasma pneumonia, Chlamydophila pneumonia, West Nile, Borrelia and Toscana was negative. CSF bacterial, mycobacterial and micologic cultures were negative. Ceftriaxone and ampicillin were discontinued 1 day after admission, as well as acyclovir after CSF HSV1 and 2 and VZV PCR came back negative.

Three days after admission, LP was repeated, revealing 330 cells (98.5\% mononuclear), glucose $56 \mathrm{mg} / \mathrm{dL}$ with elevated protein level $(1.95 \mathrm{~g} / \mathrm{L})$. Autoimmune study and serologic tests for HIV, hepatitis, syphilis, CMV, EBV, herpes simplex $1 \& 2$, Borrelia, toxoplasmosis, Chlamydia pneumonia and varicella were also negative. Repeated ECK was normal and the patient was discharged on day 10 with levetiracetam 1,000 $\mathrm{mg}$ twice a day. At this time, HaNDL had already been considered and the patient was counseled and oriented to follow-up by neurology.
Two days after discharge, he developed a similar clinical picture, with dizziness, paresthesia in the right upper arm, dysarthria and headache. He was brought to the ED and recovered completely $30 \mathrm{~min}$ after admission. Neurological exam was normal. He repeated LP that was similar to the previous one, the opening pressure was normal and MRI did not reveal any of the previous changes. Levetiracetam was slowly tapered and the patient remained symptom-free at 12 weeks of followup. Given the presentation (clinical picture, imaging and CSF studies), the subsequent similar and self-limited episode and having excluded other causes, a final diagnosis of HaNDL was made.

\section{Discussion}

With this clinical case, the authors pretend to draw attention to this entity that is frequently underdiagnosed and can lead to high cost imaging studies and non-innocuous interventions such as broad-spectrum antibiotics and thrombolysis.

As for the patient presented, it is most frequently seen in 
young adults ( 15 - 40 years) and is characterized by recurrent episodes ( 1 to more than 15) of headache and neurological deficits that last from a few hours to 3 days and are separated by symptom-free intervals [2]. Unlike migraine, it has male predominance, a lack of personal or familiar history of migraine and a prodrome of viral infection in about one-third of the cases [4].

Etiology of HaNDL is not yet fully understood. Most studies suggest infectious, vascular and autoimmune mechanisms $[5,6]$. Currently, the main hypothesis is that this process is secondary to an activation of the immune system due to an infectious/inflammatory process, which activates the trigeminovascular system, leading to the development of focal neurological symptoms by a mechanism similar to the neuronal spreading depression seen in migraines with aura $[7,8]$.

The most common transient neurological findings are hemisensory disturbances affecting mainly the face and distal upper $\operatorname{limb}(78 \%)$, followed by motor aphasia and motor disorders $[1$, $2,8]$. In more than $80 \%$, the deficits are confined to the dominant hemisphere [9]. Trunk and leg involvement, visual symptoms, acute confusional state and epileptic seizures are rare [1].

An LP is essential for diagnosis, since CSF lymphocytic pleocytosis (range $10-760$ cells $/ \mathrm{mm}^{3}$ ) is a constant feature [8]. Elevated CSF opening pressure can be present in up to half of the patients and CSF protein is usually elevated with normal glucose $[1,9]$. Bacterial, viral, fungal and serological studies of the CSF must be negative [1,2]. Our patient was submitted to three LPs that showed progressive improvement as expected due to the clinical resolution.

Our patients' MRI revealed a decreased and delayed perfusion to the entire left hemisphere that resolved in a few days. Though normal neuroimaging is usually present, non-specific abnormalities are occasionally seen $[3,10]$. As previously described in HaNDL, CT or MRI perfusion techniques may show temporary global hemispheric hypoperfusion that correlated with neurologic deficits in acutely symptomatic patients (Fig. 1).

Given the self-limited nature of the disease and favorable outcome (with all patients recovering by 3 months), therapeutic interventions other than symptomatic treatment of the headache are largely unnecessary [8].

\section{Conclusion}

HaNDL is one of the most frequent stroke mimics and probably not as rare as commonly thought. After exclusion of other significant pathologies that require specific therapeutic measures, in a patient with headache accompanied by recurrent neurological disorder episodes, CSF pleocytosis and normal imaging tests, HaNDL syndrome should always be considered. It has a good prognosis since this monophasic, self-limiting condition runs its course usually within a few months from onset, without any neurological sequelae.

\section{Conflict of Interest}

None.

\section{References}

1. Gomez-Aranda F, Canadillas F, Marti-Masso JF, DiezTejedor E, Serrano PJ, Leira R, Gracia M, et al. Pseudomigraine with temporary neurological symptoms and lymphocytic pleocytosis. A report of 50 cases. Brain. 1997;120 (Pt 7):1105-1113.

2. Cifelli A, Vaithianathar L. Syndrome of transient Headache and Neurological Deficits with cerebrospinal fluid Lymphocytosis (HaNDL). BMJ Case Rep. 2011;2011

3. Pettersen JA, Aviv RI, Black SE, Fox AJ, Lim A, Murray BJ. Global hemispheric CT hypoperfusion may differentiate headache with associated neurological deficits and lymphocytosis from acute stroke. Stroke. 2008;39(2):492493.

4. Tada Y, Negoro K, Abe M, Ogasawara J, Kawai M, Morimatsu M. A patient of migraine-like headache with amnesia, pleocytosis and transient hypoperfusion of cerebral blood flow. Intern Med. 2005;44(7):743-746.

5. Nelson S. Confusional State in HaNDL Syndrome: Case Report and Literature Review. Case Rep Neurol Med. 2013;2013:317685.

6. Chapman KM, Szczygielski BI, Toth C, Woolfenden A, Robinson G, Snutch TP, Spacey SD. Pseudomigraine with lymphocytic pleocytosis: a calcium channelopathy? Clinical description of 10 cases and genetic analysis of the familial hemiplegic migraine gene CACNA1A. Headache. 2003;43(8):892-895.

7. Soto-Insuga V, Lopez-Villanueva L, Rodrigo M, Mois Aroyo I, Losada R, Soriano-Guillen L. [Confusion as a presentation symptom of pseudomigraine with pleocytosis in a paediatric patient]. An Pediatr (Barc). 2014;80(6):394-398.

8. Oban A, Shugaiv E, Tuzun E. Syndrome of headache accompanied with transient neurologic deficits and cerebrospinal fluid lymphocytosis. Arch Neuropsychiatr. 2013;50:52-55.

9. Kablan Y, Kamisli O, Celik H. HaNDL Syndrome Presenting During Pregnancy: A Case Report and Review of the Literature. J Neurol Sci. 2013;333:517-518.

10. Vallet AE, Desestret V, Tahon F, Cho TH, Nighoghossian N. Acute perfusion MR imaging in a HaNDL-like syndrome. Cerebrovasc Dis. 2010;29(1):98-100. 